\title{
Histone deacetylase 5 regulates glucose uptake and insulin action in muscle cells
}

\author{
Suryaprakash Raichur, Song Hooi Teh, Kenji Ohwaki, Vidhi Gaur ${ }^{1}$, Yun Chau Long ${ }^{2}$, \\ Mark Hargreaves ${ }^{3}$, Sean L McGee ${ }^{1}$ and Jun Kusunoki
}

\author{
Lilly Singapore Centre for Drug Discovery, 8A Biomedical Grove \#02-05, Immunos 138648, Singapore \\ ${ }^{1}$ Metabolic Research Unit, School of Medicine, Deakin University, Pigdons Road, Waurn Ponds, Geelong, Victoria 3217, Australia \\ ${ }^{2}$ Department of Biochemistry, Yong Loo Lin School of Medicine, National University of Singapore, Singapore \\ ${ }^{3}$ Department of Physiology, The University of Melbourne, Parkville, Victoria, Australia \\ (Correspondence should be addressed to S L McGee; Email: sean.mcgee@deakin.edu.au; J Kusunoki; Email: jun_kusunoki@lilly.com)
}

\begin{abstract}
The class lla histone deacetylases (HDACs) act as transcriptional repressors by altering chromatin structure through histone deacetylation. This family of enzymes regulates muscle development and phenotype, through regulation of muscle-specific genes including myogenin and MyoD (MYOD1). More recently, class lla HDACs have been implicated in regulation of genes involved in glucose metabolism. However, the effects of HDAC5 on glucose metabolism and insulin action have not been directly assessed. Knockdown of HDAC5 in human primary muscle cells increased glucose uptake and was associated with increased GLUT4 (SLC2A4) expression and promoter activity but was associated with reduced GLUT1 (SLC2A1) expression. There was no change in PGC-1 $\alpha$ (PPARGC1A) expression. The effects of HDAC5 knockdown on glucose metabolism were not due to alterations in the initiation of differentiation, as knockdown of $H D A C 5$ after the onset of differentiation also resulted in increased glucose uptake and insulin-stimulated glycogen synthesis. These data show that inhibition of HDAC5 enhances metabolism and insulin action in muscle cells. As these processes in muscle are dysregulated in metabolic disease, HDAC inhibition could be an effective therapeutic strategy to improve muscle metabolism in these diseases. Therefore, we also examined the effects of the pan HDAC inhibitor, Scriptaid, on muscle cell metabolism. In myotubes, Scriptaid increased histone 3 acetylation, GLUT4 expression, glucose uptake and both oxidative and non-oxidative metabolic flux. Together, these data suggest that HDAC5 regulates muscle glucose metabolism and insulin action and that HDAC inhibitors can be used to modulate these parameters in muscle cells.
\end{abstract}

Journal of Molecular Endocrinology (2012) 49, 203-211

\section{Introduction}

The class IIa histone deacetylases (HDACs) act as transcriptional repressors by altering chromatin structure to a state that impairs transcription ( $\mathrm{Li}$ et al. 2007). This primarily occurs through deacetylation of histone lysine residues, which modifies the spatial relationship between histones and DNA (Li et al. 2007). This ultimately restricts initiators of transcription from accessing gene promoter regions, resulting in transcriptional repression ( $\mathrm{Li}$ et al. 2007). The class IIa HDACs consist of isoforms 4, 5, 7 and 9 and are highly enriched in skeletal muscle, the heart and the brain (McKinsey et al. 2001). These transcriptional repressors act by associating with DNA binding transcription factors, such as the myocyte enhancer factor 2 (MEF2) to repress specific promoter regions (Lu et al. 2000). Loss-of-function animal models have shown that this family of enzymes regulates processes such as muscle development and phenotype (Potthoff et al. 2007), skeletogenesis (Vega et al. 2004), vascular integrity (Chang et al. 2006) and stress-induced cardiac hypertrophy (Chang et al. 2004). At a cellular level, perhaps the most characterised functional role of the class IIa HDACs is regulation of muscle differentiation (Lu et al. 2000, McKinsey et al. 2000, Dressel et al. 2001, Zhang et al. 2001, 2002). This principally occurs through repression of $M E F 2$ that regulates the myogenin and MyoD promoters and hence suppresses their expression (Deng et al. 2005, Micheli et al. 2005). Inhibition of HDAC function increases myogenin and $M y o D$ (MYOD1) expression and is sufficient to initiate the differentiation process that converts myoblasts to myotubes (Deng et al. 2005, Micheli et al. 2005). The class IIa HDACs are primarily regulated by phosphorylation-dependent nuclear export (McKinsey et al. 2000). Their phosphorylation provides binding sites for the 14-3-3 chaperone proteins, which export HDACs out of the nucleus via a CRM-1-dependent mechanism, which is sufficient to derepress the genes that they regulate (McKinsey et al. 2000). We have recently found that the AMP-activated protein kinase (AMPK) is a HDAC5 
kinase (McGee et al. 2008). AMPK is a cellular energy gauge, which is activated by alterations in the cellular AMP to ATP ratio and by hormones that signal low energy status (Kahn et al. 2005). In skeletal muscle, AMPK is thought to control metabolism not only by acute regulation of metabolic pathways but also by transcriptional control of these processes (McGee \& Hargreaves 2010). Indeed, we have shown that phosphorylation of HDAC5 by AMPK increases GLUT4 (SLC2A4) gene expression (McGee et al. 2008). This suggests that in addition to their role in repressing muscle differentiation, the class IIa HDACs could also have an important role in muscle glucose metabolism. Supporting this are experiments showing that cardiac expression of mutant HDAC5 that cannot be phosphorylated suppressed numerous metabolic genes, including those involved in glucose, fatty acid and oxidative metabolism (Czubryt et al. 2003). Together, these data suggest that HDAC5 could be a central regulator of metabolic genes. Although we have shown that HDAC5 regulates the GLUT4 gene, the functional effects of HDAC5 on glucose metabolism have not been directly assessed in skeletal muscle cells. Furthermore, the role of HDAC5 in insulin action has also not been assessed. Therefore, the purpose of this study was to examine whether knockdown of $H D A C 5$ regulates glucose uptake and insulin action in muscle cells and to determine whether this is independent of its effects on the initiation of differentiation. Furthermore, in proofof-principle experiments, we also sought to determine whether pharmacological HDAC inhibition can regulate these same processes, using the hydroxamate broad-spectrum class I and class II HDAC inhibitor Scriptaid. As therapies aimed at restoring metabolic homeostasis in states of overnutrition will likely have to increase cellular energy expenditure (Anderson et al. 2009), we also examined myotube bioenergetics and metabolic flux in response to Scriptaid treatment.

\section{Materials and methods}

\section{Reagents}

DMEM, Opti-MEM, fetal bovine serum (FBS), penicillin/ streptomycin (pen/strep), TRIzol, High-Capacity cDNA RT Kit and Lipofectamine LTX were from Invitrogen. Human myoblasts and myoblast media were from Lonza (Basel, Switzerland). Delta 8.9 and vsv-g plasmids were from Research Biolabs (Singapore) and pLKO.1 shRNA plasmids, horse serum (HS) and Scriptaid were from Sigma. RNeasy Mini Kits were purchased from Qiagen. TaqMan Universal PCR Master Mix and TaqMan probes for HDAC5 (Mm00515941_g1), GLUT4 (Mm01245507_g1), GLUT1 (Mm01192270_m1), MyoD (Mm00440387_m1), myogenin (Mm00446195_g1),
PGC-1 $\alpha$ (PPARGC1A) (Mm00447187_g1) and 18S (Hs999999901_s1) were from Applied Biosystems. Seahorse XF analyser culture plates and assay cartridges were from Seahorse Bioscience (Bellerica, MA, USA).

\section{Lentivirus production}

A shRNA sequence recognising bases 1324-1345 (CCATCGTGAGAATGGCTTTA) of human HDAC5 cDNA (which shows $100 \%$ homology to mouse) in the pLKO.1 vector, or the empty pLKO.1 vector (control), were transfected with delta 8.9 and vsv-g plasmids at a ratio of 10:9:1 into HEK293-LentiX cells using Lipofectamine LTX at $3 \cdot 75 \mu \mathrm{l} / \mu \mathrm{g}$ of DNA. Transfected cells were incubated at $37^{\circ} \mathrm{C}$ for $18 \mathrm{~h}$ before changing media to the target cell culture media's type including $10 \mathrm{mM}$ sodium butyrate and were incubated for a further $8 \mathrm{~h}$, before withdrawal of the sodium butyrate by media change. Cells were incubated overnight before collecting the viral supernatant. Media were replaced and the viral supernatant again collected $24 \mathrm{~h}$ later. All supernatants were filtered $(0.45 \mu \mathrm{m})$ and stored at $-80^{\circ} \mathrm{C}$.

\section{Cell culture}

HEK293-LentiX, C2C12 myoblast and L6 myoblast cells were maintained in high-glucose DMEM supplemented with $10 \% \mathrm{FBS}$ and $1 \%$ pen/strep. Human primary myoblasts were maintained in Human Myoblast Media supplemented with $10 \%$ FBS and $1 \%$ pen/strep. All myoblast cell lines were induced to differentiate to myotubes at confluence by replacing $10 \%$ FBS with $2 \%$ HS. All cultures were maintained in a humidified incubator at $37^{\circ} \mathrm{C}$ with $5 \% \mathrm{CO}_{2}$. C2C12 and human primary myoblasts were infected with lentiviral supernatant at $3000 \mathrm{cell} / \mathrm{cm}^{2}$. C2C12 myoblasts with stable knockdown of HDAC5 were generated by selection with $1 \mu \mathrm{g} / \mathrm{ml}$ puromycin for four passages (14 days) before experiments. Upon confluence, myoblasts were induced to differentiate to myotubes by exchanging media serum from $10 \%$ FBS to $2 \%$ HS. Scriptaid treatment was performed for $48 \mathrm{~h}$ at day 4 post-differentiation.

\section{Real time RT-PCR}

Total RNA was isolated by collecting cells in TRIzol and taking the upper aqueous phase after incubation with chloroform. An equal volume of $70 \%$ ethanol was added and RNA was extracted using the RNeasy Mini Kit columns. RNA was eluted in $50 \mu$ l RNase-free water and the concentration of RNA was estimated by spectrophotometery. cDNA $(2 \mu \mathrm{g})$ was synthesised using the High-Capacity cDNA RT Kit. Gene expression was determined using TaqMan probes for HDAC5, 
GLUT4, GLUT1 (SLC2A1), MyoD, myogenin, PGC-1 $\alpha$ and $18 S$ and TaqMan Universal PCR Master Mix. Gene expression levels were determined using the $\Delta \Delta C T$ method using the housekeeping gene 18S. $18 \mathrm{~S}$ expression did not change in any of the interventions tested (data not shown).

\section{GLUT4 reporter assay}

Each 96-well plate of control and stable HDAC5 k/d C2C12 myoblasts and human primary myoblasts $(\sim 60 \%$ confluence) were transfected with a total of $\sim 2 \cdot 0-3 \cdot 0 \mu \mathrm{g}$ DNA per plate using the Lipofectamine (Invitrogen)-mediated transfection. Procedure was followed as per the manufacturer's instructions. Briefly, pSGG-Prom reporter vector (Switchgear Genomics, Menlo Park, USA) containing $~ 900$ bp human Glut4 promoter was transfected with or without HDAC5 shRNA. The transfection efficiencies were normalised with Renilla luciferase vector. The culture medium was changed $24 \mathrm{~h}$ later and upon reaching $100 \%$ confluency cells were differentiated for 2 days. Subsequently, cells were harvested for the dual luciferase assay. Fold activation was expressed relative to activity obtained after co-transfection of the promoter-reporter and control shRNA vectors only, arbitrarily set at 1 .

\section{Glucose uptake}

Cell media from myoblast and myotube cultures in 96-well plates were removed and cells were washed twice with Krebs-Ringer HEPES buffer $(130 \mathrm{mM} \mathrm{NaCl}$, $5 \mathrm{mM} \mathrm{KCl}, 2 \cdot 5 \mathrm{mM} \mathrm{CaCl}_{2}, 20 \mathrm{mM}$ HEPES, $1.2 \mathrm{mM}$ $\mathrm{MgSO}_{4}, 1 \cdot 2 \mathrm{mM} \mathrm{KH}_{2} \mathrm{PO}_{4}$ and $0 \cdot 1 \% \mathrm{BSA}$ ), before being incubated in this buffer for $60 \mathrm{~min}$ at $37^{\circ} \mathrm{C}$ with $5 \% \mathrm{CO}_{2}$. Cells were then incubated with labelled deoxy-[ $\left.\mathrm{H}^{3}\right]$-D-glucose $(0 \cdot 25 \mu \mathrm{Ci} ; 5 \mu \mathrm{M})$ and unlabelled 2-deoxy-D-glucose $(2 \mathrm{mM})$ for $15 \mathrm{~min}$ at $37^{\circ} \mathrm{C}$ with $5 \% \mathrm{CO}_{2}$ before being washed twice with Krebs-Ringer HEPES buffer. Cells were solubilised with $30 \mu \mathrm{l}$ $0 \cdot 1 \%$ SDS and $20 \mu \mathrm{l}$ was transferred to an Isoplate-96 Microplate. Scintillation $(100 \mu \mathrm{l})$ cocktail was added to each well and incubated at room temperature for $2 \mathrm{~h}$ before measurement with a Beta Counter. The remaining $10 \mu \mathrm{l}$ cell lysate were used to determine protein concentration, with deoxy-[ $\left.\mathrm{H}^{3}\right]$-D-glucose normalised to protein content.

\section{Glycogen synthesis}

Assays were performed on myotubes after 4 days of differentiation. Differentiation media were removed and myotubes were serum starved (DMEM and $0.2 \% \mathrm{BSA}$ ) for $3 \mathrm{~h}$ before the addition of $50 \mathrm{nM}$ insulin or vehicle and $\left[\mathrm{U}_{-} \mathrm{C}^{14}\right]$-D-glucose $(1 \mu \mathrm{Ci})$ followed by further incubation for $120 \mathrm{~min}$ in the culture incubator.
Cells were washed twice with PBS before being lysed in $300 \mu \mathrm{l} 1 \mathrm{M} \mathrm{NaOH}$. Lysates were left to incubate overnight at room temperature, followed by the addition of $10 \mathrm{mg} / \mathrm{ml}$ cold glycogen $(1: 1, \mathrm{v} / \mathrm{v})$ and $100 \%$ ethanol $(1: 1, \mathrm{v} / \mathrm{v})$. Samples were incubated at $-20{ }^{\circ} \mathrm{C}$ for $30 \mathrm{~min}$ before being spun in a centrifuge at $12000 \mathrm{~g}$ for $10 \mathrm{~min}$ at $4{ }^{\circ} \mathrm{C}$. The pellet was solubilised with $100 \mu \mathrm{l}$ deionised water and $20 \mu \mathrm{l}$ were transferred to an Isoplate-96 Microplate. Scintillation $(100 \mu \mathrm{l})$ cocktail was added to each well and incubated at room temperature for $2 \mathrm{~h}$, before measurement with a Beta Counter.

\section{Western blotting}

Cells were collected in RIPA buffer and the total protein content of cell lysates was determined using the DC Protein Assay Kit (Bio-Rad). Protein $(30 \mu \mathrm{g})$ was mixed with NuPAGE LDS Sample Buffer (Invitrogen) and was separated by SDS-PAGE. Protein was transferred to membranes using the iBlot System (Invitrogen), blocked with 5\% BSA in Tris-buffered saline with $0.25 \%$ Tween 20 (TBST) for $30 \mathrm{~min}$ at RT. Membranes were exposed to primary antibodies overnight at $4{ }^{\circ} \mathrm{C}$, washed in TBST, exposed to secondary antibodies for $60 \mathrm{~min}$ at RT and washed again. Antibody binding was visualised using the Li-Cor Odyssey System.

\section{Bioenergetics and mitochondrial function assays}

Bioenergetic and mitochondrial function analyses were performed using the Seahorse XF24 Extracellular Flux Analyser (Seahorse Bioscience). L6 myoblasts were seeded into 24-well Seahorse V7 plates at $2.5 \times 10^{4}$ cells/well. The following day, cells were differentiated to myotubes by serum withdrawal. After 4 days of differentiation, myotubes were treated with $0 \cdot 1$ or $1 \mu \mathrm{M}$ Scriptaid, or vehicle (PBS and 10\% DMSO) for $60 \mathrm{~min}$, twice per day, for 2 days. Myotubes were assayed on day 6 of differentiation, $16 \mathrm{~h}$ after the final Scriptaid treatment. Before assay, cells were washed twice with assay running media (unbuffered DMEM, $25 \mathrm{mM}$ glucose, $1 \mathrm{mM}$ glutamine and $1 \mathrm{mM}$ sodium pyruvate), before being resuspended in $675 \mu \mathrm{l}$ running media. Cells were equilibrated in a non- $\mathrm{CO}_{2}$ incubator for $60 \mathrm{~min}$ before assay. The assay protocol consisted of repeated cycles of 4-min mix, 2-min wait and 2-min measurement periods, with oxygen consumption rate (OCR) and extracellular acidification rate (ECAR) measured simultaneously through each measurement period by excitation of fluorophores for $\mathrm{O}_{2}$ and $\mathrm{H}^{+}$. This gives measurement of oxidative and non-oxidative metabolism respectively. Basal energetics were established after three of these cycles, followed by sequential exposure of the ATP synthase inhibitor oligomycin, the proton ionophore carbonyl cyanide $p$-trifluoromethoxyphenylhydrazone 
(FCCP) and the complex III inhibitor antimycin A, all to a final concentration of $1 \mu \mathrm{M}$. These compounds were introduced to the cell media by the Seahorse injection system. Three mix, wait and measurement cycles separated each compound injection. Respiration due to ATP turnover was determined by subtracting the mean respiration values following oligomycin addition from basal respiration rates. Uncoupled respiration was determined by subtracting the mean respiration rate following antimycin A addition from that following oligomycin addition. Maximal respiratory capacity was determined by respiration following FCCP addition. Spare respiratory capacity was established by subtracting basal respiration rate from maximal respiration. All calculations were performed on values obtained in each well and all treatment conditions were analysed as ten replicates, over at least two independent experiments and data were pooled to give average values for each treatment.

\section{Statistical analysis}

All values are reported as mean \pm s.E.M. and were evaluated for statistically significant differences using $t$-tests, ANOVA or two-way ANOVA where appropriate. Differences between groups were considered statistically significant where $P<0 \cdot 05$.

\section{Results}

\section{Knockdown of HDAC5 increases glucose uptake and regulates GLUT expression in human primary myotubes}

We have previously shown that HDAC5 regulates the GLUT4 gene (McGee et al. 2008). To assess the role of HDAC5 on metabolism and insulin action, we infected human primary myoblasts with a lentivirus expressing HDAC5 shRNA. Myoblasts were then differentiated to myotubes for 4 days before analysis of gene expression and glucose uptake. We achieved $\sim 90 \%$ knockdown of HDAC5 gene expression following infection of human primary myoblasts with the HDAC5 shRNA lentivirus (Fig. 1A). HDAC5 knockdown significantly increased basal glucose uptake (Fig. 1B), which was associated with enhanced expression of the glucose transport isoform 4 (GLUT4) gene (Fig. 1C). To determine whether the regulation of GLUT4 expression was associated with regulation of the GLUT4 promoter, gene reporter assays were performed using a plasmid expressing the luciferase gene driven by $\sim 900 \mathrm{bp}$ of the GLUT4 promoter. A titrated knockdown of HDAC5 resulted in reciprocal increases in GLUT4 promoter activity (Fig. 1D). We also assessed GLUT1 gene expression, which is a contributor to basal glucose
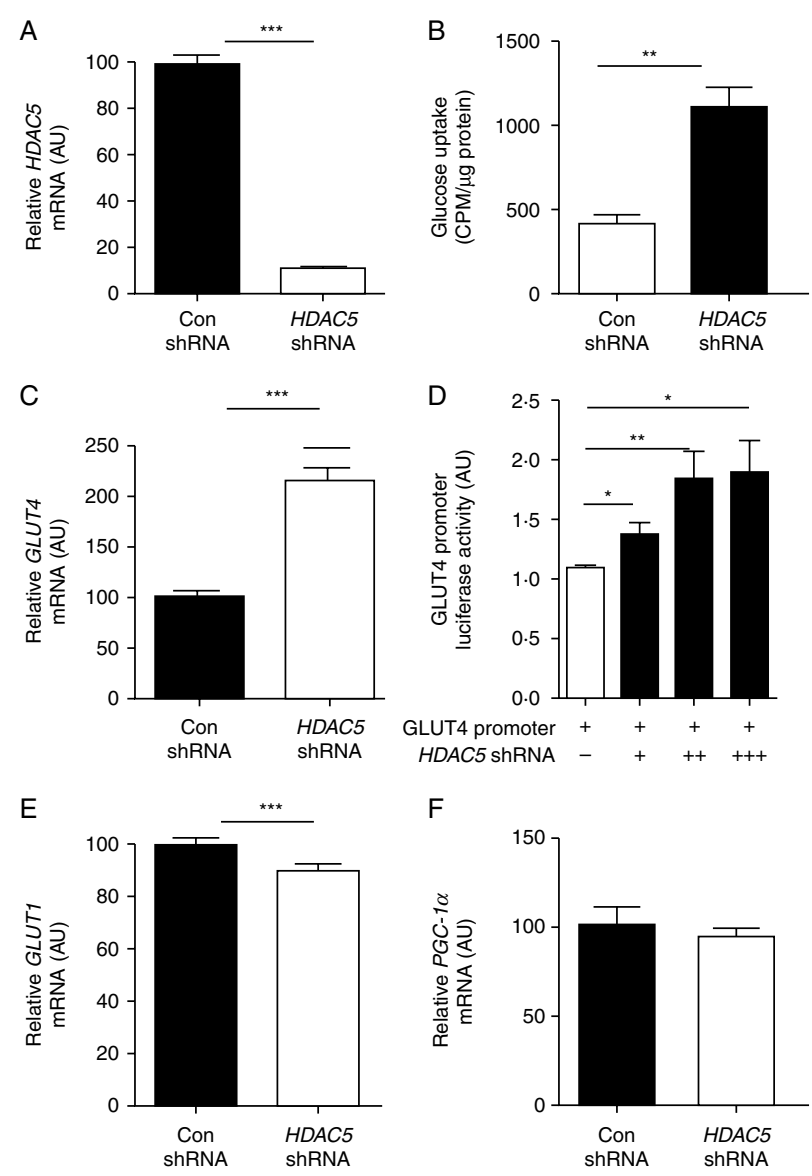

Figure 1 Knockdown of HDAC5 increases glucose uptake and regulates GLUT expression in human primary myotubes (A) HDAC5 mRNA expression in control and HDAC5 knockdown human primary myotubes; $(\mathrm{B})$ glucose uptake in control and HDAC5 knockdown human primary myotubes; (C) GLUT4 mRNA in control and $H D A C 5$ knockdown human primary myotubes; (D) GLUT4 promoter activity in control and HDAC5 knockdown human primary myotubes; (E) GLUT1 mRNA in control and HDAC5 knockdown human primary myotubes; and (F) $P G C-1 \alpha$ mRNA in control and HDAC5 knockdown human primary myotubes. Data are expressed as mean \pm S.E.M. $n=3-6$ per group. ${ }^{\star} P<0.05,{ }^{\star \star} P<0.01$ and ${ }^{\star \star \star} P<0.001$ vs control knockdown.

uptake, and found that HDAC5 knockdown resulted in a small but statistically significant decrease in GLUT1 mRNA (Fig. 1E). We also assessed the expression of $P G C-1 \alpha$, which is thought to be a central regulator of metabolic gene expression (Lin 2009) and a target of HDAC5 (Czubryt et al. 2003), and found no change in $P G C-1 \alpha$ gene expression (Fig. 1F).

\section{Enhanced glucose uptake is independent of differentiation and HDAC5 knockdown enhances insulin action}

The differentiation of myoblasts to myotubes is known to increase cellular metabolism and metabolic capacity 
(Franko et al. 2008). To determine whether the increased basal glucose uptake was due to alterations in the initiation of differentiation regulated by HDAC5, we infected human myoblasts with the HDAC5 shRNA lentivirus $48 \mathrm{~h}$ after the onset of differentiation and then assessed basal glucose uptake $48 \mathrm{~h}$ after infection. Glucose uptake was again enhanced following HDAC5 knockdown using this protocol (Fig. 2A). To assess whether HDAC5 regulates insulin action, we also performed basal and insulin-stimulated glycogen synthesis in human primary myotubes using this same differentiation/knockdown procedure. HDAC5 knockdown had a small but significant effect on basal glycogen synthesis; however, insulin-stimulated glycogen synthesis was increased by $46 \%$ in HDAC5 knockdown cells, suggesting enhanced insulin action (Fig. 2B).
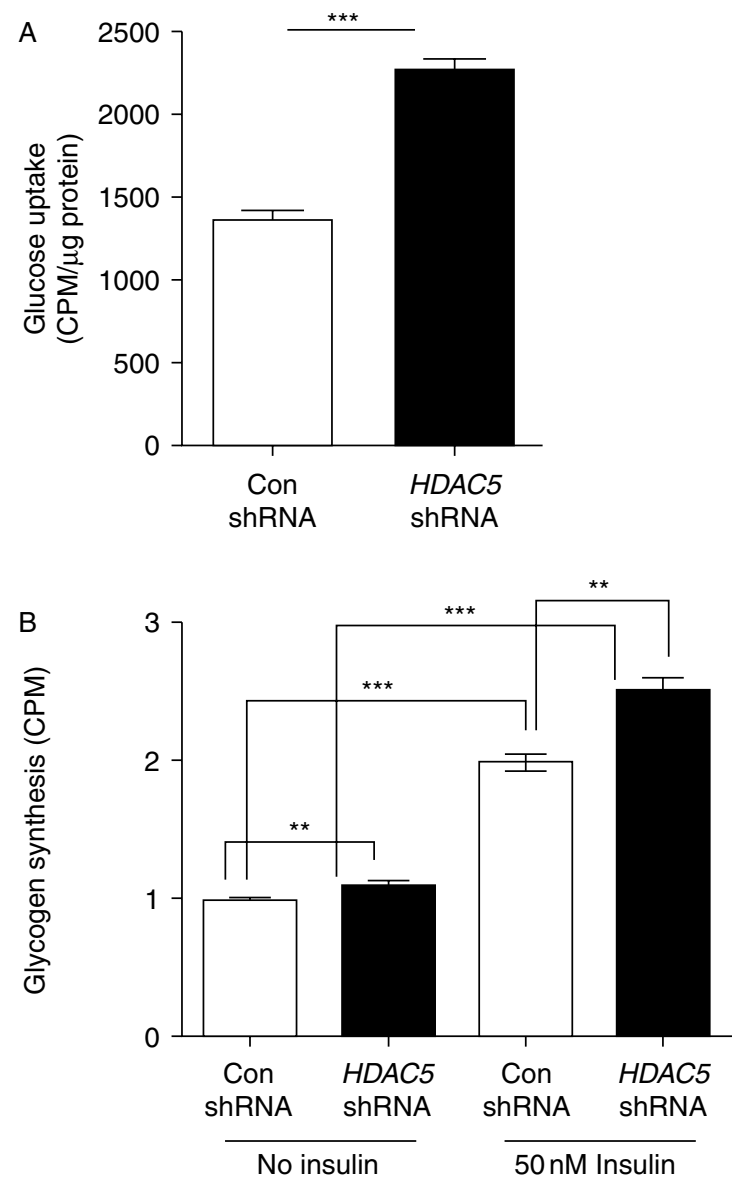

Figure 2 Enhanced glucose uptake is not due to altered differentiation and HDAC5 knockdown enhances insulin action. (A) Glucose uptake in control and HDAC5 knockdown human primary myotubes, infected after the initiation of differentiation and (B) basal and insulin-stimulated (50 nM) glycogen synthesis in control and HDAC5 knockdown human primary myotubes, infected after the initiation of differentiation. Data are expressed as mean \pm S.E.M. $n=6-8$ per group. ${ }^{\star \star} P<0.01$ and ${ }^{\star \star \star} P<0.001$.
The effects of HDAC5 knockdown on GLUT4 promoter activity and glucose uptake are consistent in two different muscle cell types

To determine whether the observed effects of HDAC5 knockdown on GLUT4 regulation and glucose uptake were specific for human primary muscle cells, we assessed GLUT4 promoter activity and glucose uptake in $\mathrm{C} 2 \mathrm{C} 12$ myotubes following HDAC5 knockdown. Infection of C2C12 myoblasts with HDAC5 shRNA lentivirus before differentiation resulted in $62 \%$ knockdown of HDAC5 in myotubes (Fig. 3A). Knockdown of HDAC5 also increased GLUT4 promoter activity (Fig. 3B) and glucose uptake (Fig. 3C) in C2C12 myotubes. These data show that HDAC5 is a regulator of GLUT4 and glucose uptake in two different muscle cell types.

\section{Scriptaid, a broad-spectrum HDAC inhibitor, also regulates GLUT4 gene expression, glucose uptake and mitochondrial function}

The data presented to date show that inhibition of HDAC5 can enhance glucose metabolism and insulin action in muscle cells. Therefore, we also examined the effects of the broad-spectrum HDAC inhibitor, Scriptaid, on metabolism. Forty-eight hours of Scriptaid exposure increased histone 3 acetylation in a dose-dependent manner in L6 myotubes (Fig. 4A). This was associated with significant increases in GLUT4 expression at Scriptaid concentrations of $0.5 \mu \mathrm{M}$ and higher (Fig. 4B). Incubation of L6 myotubes with $1 \mu \mathrm{M}$ Scriptaid for $48 \mathrm{~h}$ also increased basal glucose uptake (Fig. 4C), with no change in GLUT1 gene expression (data not shown). We also assessed the ability of Scriptaid to alter cellular bioenergetics using the Seahorse XF analyser. Myotubes were exposed to Scriptaid or vehicle for $1 \mathrm{~h}$, twice per day for 2 days before assay. This protocol was used to mimic potential drug treatment and pharmacokinetics in vivo. Incubation of myotubes with $0 \cdot 1 \mu \mathrm{M}$ Scriptaid increased basal OCR, while $1 \mu \mathrm{M}$ Scriptaid increased both basal OCR and ECAR (Fig. 4D), suggesting that Scriptaid increases cellular energy expenditure. To determine the metabolic parameter(s) contributing to the increase in basal oxidative metabolism, we also performed mitochondrial function tests on these cells. Scriptaid increased basal ATP turnover (Table 1), without any change in uncoupled respiration, which are the two parameters contributing to basal OCR. Scriptaid also increased maximal and spare respiratory capacity, while $1 \mu \mathrm{M}$ Scriptaid also increased nonmitochondrial oxygen consumption (Table 1). 


\section{Discussion}
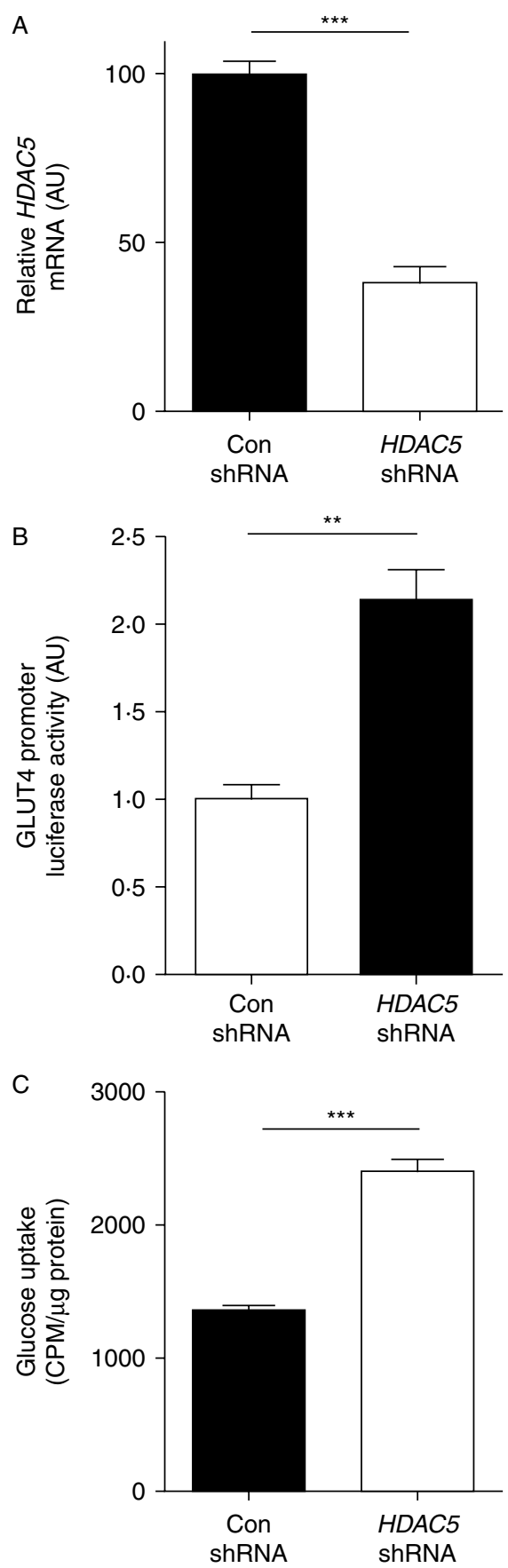

Figure 3 Knockdown of HDAC5 increases GLUT4 promoter activity and glucose uptake in $\mathrm{C} 2 \mathrm{C} 12$ myotubes. (A) HDAC5 mRNA expression in control and HDAC5 knockdown C2C12 myotubes; (B) GLUT4 promoter activity in control and HDAC5 knockdown C2C12 myotubes; and (C) glucose uptake in control and HDAC5 knockdown human primary myotubes; data are expressed as mean \pm S.E.M. $n=3-6$ per group. ${ }^{* \star} P<0.01$ and ${ }^{* \star \star} P<0.001$ vs control knockdown.
We have previously shown that HDAC5 represses the GLUT4 gene (McGee et al. 2008). However, to date, no study has examined whether members of the class IIa HDACs actually regulate glucose metabolism in skeletal muscle cells. Our data show that knockdown of HDAC5 is sufficient to increase glucose uptake in both human primary myotubes and mouse C2C12 myotubes. This was associated with increased GLUT4 promoter activity in the two cell types examined. Whether the increase in glucose uptake with $H D A C 5$ knockdown is solely due to enhanced GLUT4 expression remains to be determined; however, HDAC5 has been shown to repress a number of genes involved in glucose metabolism, including hexokinase II and glycogen phosphorylase (Czubryt et al. 2003). This study also suggests that the effect of HDAC5 knockdown on glucose uptake occurs independently of any increase in $P G C-1 \alpha$ expression. This is an interesting finding given that the class IIa HDACs are thought to regulate the PGC- $1 \alpha$ promoter and that many of the muscle phenotypic effects of the class IIa HDACs have been ascribed to regulation of PGC- $1 \alpha$ (Czubryt et al. 2003). However, HDAC5 is known to directly repress transcription factors such as MEF2 (Lu et al. 2000), serum response factor (SRF; Xing et al. 2006) and the nuclear factor of activated T-cells (Dai et al. 2005), which are also known to exert phenotypic effects in muscle. Data from this study also show that HDAC5 knockdown enhances insulin action. As cultured myotubes show limited insulin responsiveness as assayed by glucose uptake, we assessed insulin-stimulated glycogen synthesis in these cells. Using this method, we found that the fold increase in insulin action was $46 \%$ greater in $H D A C 5$ knockdown cells. It remains to be determined whether HDAC5 knockdown enhances insulin action per se, or whether it regulates the expression of rate-limiting enzymes in this pathway. However, the findings that HDAC5 regulates glucose uptake and insulin action are important, as these processes are dysregulated in metabolic diseases such as obesity and type 2 diabetes (Savage et al. 2007). Therefore, targeting the class IIa HDACs in muscle could be an effective treatment strategy to normalise these defects in metabolic disease states.

With this in mind, we also tested the efficacy of the broad spectrum class I and class II HDAC inhibitor Scriptaid, in proof-of-principle experiments, to determine whether HDAC inhibition also regulates metabolic processes in myotubes. These data revealed that GLUT4 expression and glucose uptake were also increased following exposure to Scriptaid. These glucose uptake data are similar to those reported by Takigawa-Imamura et al. (2003). However, that paper showed no effect of Scriptaid on GLUT4 gene expression (Takigawa-Imamura et al. 2003), while we 

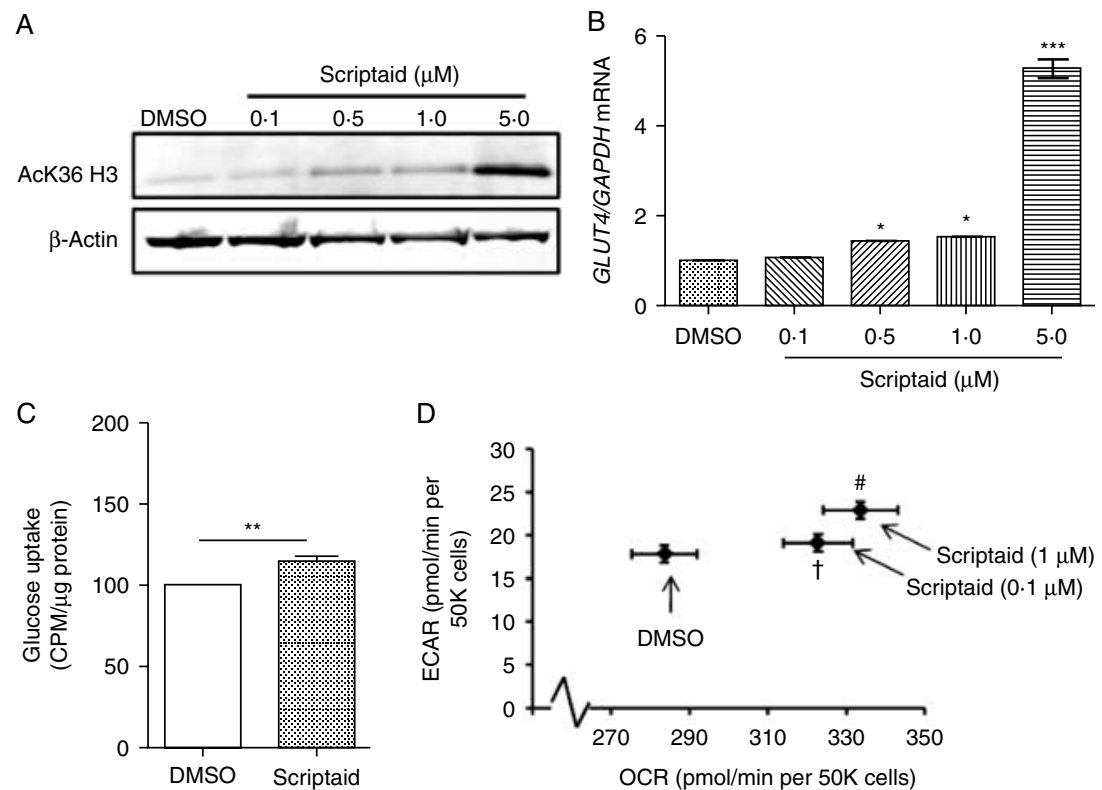

Figure 4 Scriptaid regulates GLUT4 gene expression, glucose uptake and mitochondrial function. (A) Histone 3 acetylation in response to increasing concentrations of Scriptaid for $48 \mathrm{~h}$ in L6 myotubes; (B) GLUT4 mRNA in response to increasing concentrations of Scriptaid for $48 \mathrm{~h}$ in L6 myotubes; (C) glucose uptake in response to $1 \mu \mathrm{M}$ Scriptaid for $48 \mathrm{~h}$ in L6 myotubes and (D) basal OCR and ECAR in L6 myotubes in response to multiple treatments with 0.1 and $1 \mu \mathrm{M}$ Scriptaid. Data are expressed as mean \pm S.E.M. $n=20$ per group, across two independent experiments. ${ }^{\star} P<0.05$, ${ }^{\star \star} P<0.01$ and ${ }^{\star \star \star}{ }^{*} P<0.001$ vs DMSO, ${ }^{\dagger} P<0.05$ vs DMSO for OCR and ${ }^{\#} P<0.05$ vs DMSO for OCR and ECAR.

observed a correlation between glucose uptake and GLUT4 expression. Nonetheless, these data show that HDAC inhibition studies in whole-body models of metabolic disease are warranted. Scriptaid has similar inhibitory activities towards HDAC5 $\left(\mathrm{IC}_{50}=\sim 2 \mu \mathrm{M}\right)$ and the class I HDACs $\left(\mathrm{IC}_{50}=\sim 0 \cdot 6 \mu \mathrm{M}\right.$; Bradner et al. 2010), and thus, we cannot conclude that the Scriptaid effect on metabolism is mediated entirely through HDAC5. Indeed, there is good rationale for inhibiting both HDAC5 and the class I HDACs, as the class IIa HDACs are thought not to contain intrinsic HDAC activity, but instead gain HDAC activity via recruitment of HDAC3 as part of a multi-protein repressive complex
(Fischle et al. 2002). It has been proposed that HDAC inhibitors impair class IIa activity by dissociating this repressive complex from class IIa HDACs (Bradner et al. 2010). These experiments provide the impetus to develop more specific class IIa-specific inhibitors to determine the specificity requirements for these compounds to increase GLUT4 and metabolic gene expression.

However, it is generally accepted that any therapy designed to completely resolve skeletal muscle metabolic dysregulation seen in response to the caloric excess observed in many metabolic disease states will likely need to increase basal skeletal muscle energy

Table 1 Mitochondrial function (Mt Fx) parameters in response to Scriptaid. Data are expressed as mean \pm S.E.M. $n=8$ per group

\section{Mt Fx parameter (OCR pmol/min)}

ATP turnover Uncoupled respiration Maximal respiratory capacity Spare respiratory capacity Non-mitochondrial $\mathrm{O}_{2}$ consumption

${ }^{*} P<0.05$ vs DMSO, ${ }^{\ddagger} P<0.001$ vs DMSO.

\section{Scriptaid}

\begin{tabular}{cccc}
\hline $0 \mu \mathrm{M}$ & $0 \cdot 1 \mu \mathrm{M}$ & & $1 \mu \mathrm{M}$ \\
$236 \cdot 4 \pm 6 \cdot 5$ & & & \\
$47 \cdot 2 \pm 3 \cdot 3$ & $475 \cdot 0 \pm 7 \cdot 8^{\ddagger}$ & & $283 \cdot 0 \pm 7 \cdot 7^{\ddagger}$ \\
$866 \cdot 5 \pm 37 \cdot 3$ & $1069 \cdot 8 \pm 42 \cdot 5^{\ddagger}$ & & $50 \cdot 6 \pm 2 \cdot 6$ \\
$497 \cdot 0 \pm 28 \cdot 8$ & $652 \cdot 1 \pm 33 \cdot 1^{\ddagger}$ & & $607 \cdot 5 \pm 41 \cdot 0^{\ddagger}$ \\
$86 \cdot 0 \pm 3 \cdot 2$ & $94 \cdot 9 \pm 4 \cdot 0$ & & $97 \cdot 8 \pm 3 \cdot 6^{\ddagger}$ \\
& & &
\end{tabular}


expenditure (Anderson et al. 2009). We assessed whether Scriptaid also regulated basal energy expenditure by measuring cellular OCR and ECAR using the Seahorse XF analyser. Scriptaid increased basal OCR, and at higher concentrations also increased ECAR, a proxy measure of anaerobic glycolysis. This suggests that Scriptaid can increase basal energy expenditure through increased oxidative and nonoxidative metabolism. To determine the mechanism by which Scriptaid increases oxidative metabolism, we also performed mitochondrial function tests on these cells. These analyses revealed that the increase in basal respiration was due to enhanced ATP turnover, while there was no contributing effect from uncoupled respiration. Future studies will be required to determine the mechanism resulting in higher ATP turnover. These data complement recent findings that hepatic glucose production is under the control of the class IIa HDACs (Mihaylova et al. 2011). Together, these studies highlight that further studies examining the utility of HDAC inhibition as a therapeutic strategy in the treatment of metabolic diseases such as obesity and type 2 diabetes are warranted.

Importantly, we also showed that the effects of HDAC5 knockdown on glucose uptake were independent of myoblast differentiation. As metabolic processes such as glucose uptake and mitochondrial capacity are increased throughout myogenic differentiation (Franko et al. 2008), it was possible that the increased glucose uptake observed with HDAC5 knockdown was simply due to enhanced myogenesis. However, we showed that the increase in glucose uptake was preserved when HDAC5 knockdown was performed after the initiation of myogenic differentiation. These data suggest that targeting the class IIa HDACs in fully differentiated muscle cells in vitro is effective in modulating metabolism. This is further supported by data showing that Scriptaid is able to increase metabolic parameters in fully differentiated myotubes.

In conclusion, this study shows that HDAC5 regulates glucose metabolism and insulin action in both human and mouse myotubes. We have also shown that HDAC inhibition in myotubes can increase glucose uptake and basal energy expenditure. As glucose metabolism is dysregulated in metabolic disease states such as obesity and type 2 diabetes, these data indicate that further experiments targeting HDAC5 as a therapeutic strategy for the treatment of these diseases are needed.

\section{Declaration of interest}

S R, S H T, K O, Y C L and J K were employees of Eli Lilly.

\section{Funding}

This study was funded in part by the Diabetes Australia Research Trust Viertel Award and an NHMRC Project Grant (APP1027227; to S L M). $\mathrm{S}$ L M is supported by an NHMRC Career Development Fellowship.

\section{Acknowledgements}

The authors thank Dr Jonathon D Sedgwick, Managing Director and Chief Scientific Officer, Lilly-Singapore Centre for Drug Discovery, and Dr Phillip J Larsen, Chief Scientific Officer, Diabetes Research, Eli Lilly, Indianapolis, USA, for their continued support during this study.

\section{References}

Anderson EJ, Lustig ME, Boyle KE, Woodlief TL, Kane DA, Lin CT, Price JW III, Kang L, Rabinovitch PS, Szeto HH et al. 2009 Mitochondrial $\mathrm{H}_{2} \mathrm{O}_{2}$ emission and cellular redox state link excess fat intake to insulin resistance in both rodents and humans. Journal of Clinical Investigation 119 573-581. (doi:10.1172/JCI37048)

Bradner JE, West N, Grachan ML, Greenberg EF, Haggarty SJ, Warnow T \& Mazitschek R 2010 Chemical phylogenetics of histone deacetylase inhibitors. Nature Chemical Biology 6 238-243. (doi:10.1038/nchembio.313)

Chang S, McKinsey TA, Zhang CL, Richardson JA, Hill JA \& Olson EN 2004 Histone deacetylases 5 and 9 govern responsiveness of the heart to a subset of stress signals and play redundant roles in heart development. Molecular and Cellular Biology 24 8467-8476. (doi:10.1128/MCB.24.19.8467-8476.2004)

Chang S, Young BD, Li S, Qi X, Richardson JA \& Olson EN 2006 Histone deacetylase 7 maintains vascular integrity by repressing matrix metalloproteinase 10. Cell 126 321-334. (doi:10.1016/j.cell. 2006.05.040)

Czubryt MP, McAnally J, Fishman GI \& Olson EN 2003 Regulation of peroxisome proliferator-activated receptor $\gamma$ coactivator $1 \alpha$ (PGC-1 $\alpha$ ) and mitochondrial function by MEF2 and HDAC5. PNAS 100 1711-1716. (doi:10.1073/pnas.0337639100)

Dai YS, Xu J \& Molkentin JD 2005 The DnaJ-related factor Mrj interacts with nuclear factor of activated T cells c3 and mediates transcriptional repression through class II histone deacetylase recruitment. Molecular and Cellular Biology 25 9936-9948. (doi:10.1128/MCB.25.22.9936-9948.2005)

Deng X, Ewton DZ, Mercer SE \& Friedman E 2005 Mirk/dyrk1B decrease the nuclear accumulation of class IIa histone deacetylases during skeletal muscle differentiation. Journal of Biological Chemistry 280 4894-4905. (doi:10.1074/jbc.M411894200)

Dressel U, Bailey PJ, Wang SC, Downes M, Evans RM \& Muscat GE 2001 A dynamic role for HDAC7 in MEF2-mediated muscle differentiation. Journal of Biological Chemistry 276 17007-17013. (doi:10.1074/jbc.M101508200)

Fischle W, Dequiedt F, Hendzel MJ, Guenther MG, Lazar MA, Voelter W \& Verdin E 2002 Enzymatic activity associated with class II HDACs is dependent on a multiprotein complex containing HDAC3 and SMRT/N-CoR. Molecular Cell 9 45-57. (doi:10.1016/ S1097-2765(01) 00429-4)

Franko A, Mayer S, Thiel G, Mercy L, Arnould T, Hornig-Do HT, Wiesner RJ \& Goffart S 2008 CREB-1 $\alpha$ is recruited to and mediates upregulation of the cytochrome $\mathrm{c}$ promoter during enhanced mitochondrial biogenesis accompanying skeletal muscle differentiation. Molecular and Cellular Biology 28 2446-2459. (doi:10.1128/ MCB.00980-07)

Kahn BB, Alquier T, Carling D \& Hardie DG 2005 AMP-activated protein kinase: ancient energy gauge provides clues to modern understanding of metabolism. Cell Metabolism 1 15-25. (doi:10.1016/j.cmet.2004.12.003) 
Li B, Carey M \& Workman JL 2007 The role of chromatin during transcription. Cell 128 707-719. (doi:10.1016/j.cell.2007.01.015)

Lin JD 2009 Minireview: the PGC-1 coactivator networks: chromatinremodeling and mitochondrial energy metabolism. Molecular Endocrinology 23 2-10. (doi:10.1210/me.2008-0344)

Lu J, McKinsey TA, Zhang CL \& Olson EN 2000 Regulation of skeletal myogenesis by association of the MEF2 transcription factor with class II histone deacetylases. Molecular Cell 6 233-244. (doi:10.1016/ S1097-2765(00) 00025-3)

McGee SL \& Hargreaves M 2010 AMPK-mediated regulation of transcription in skeletal muscle. Clinical Science 118 507-518. (doi:10.1042/CS20090533)

McGee SL, van Denderen BJ, Howlett KF, Mollica J, Schertzer JD, Kemp BE \& Hargreaves M 2008 AMP-activated protein kinase regulates GLUT4 transcription by phosphorylating histone deacetylase 5. Diabetes 57 860-867. (doi:10.2337/db07-0843)

McKinsey TA, Zhang CL, Lu J \& Olson EN 2000 Signal-dependent nuclear export of a histone deacetylase regulates muscle differentiation. Nature 408 106-111. (doi:10.1038/35040593)

McKinsey TA, Zhang CL \& Olson EN 2001 Control of muscle development by dueling HATs and HDACs. Current Opinion in Genetics $\mathcal{E} \mathcal{F}$ Development 11 497-504. (doi:10.1016/S0959-437X(00) 00224-0)

Micheli L, Leonardi L, Conti F, Buanne P, Canu N, Caruso M \& Tirone F 2005 PC4 coactivates MyoD by relieving the histone deacetylase 4 mediated inhibition of myocyte enhancer factor 2C. Molecular and Cellular Biology 25 2242-2259. (doi:10.1128/MCB.25. 6.2242-2259.2005)

Mihaylova MM, Vasquez DS, Ravnskjaer K, Denechaud PD, Yu RT, Alvarez JG, Downes M, Evan RM, Montminy M \& Shaw RJ 2011 Class IIa histone deacetylases are regulators of FOXO and mammalian glucose homeostasis. Cell 145 607-621. (doi:10.1016/j.cell.2011.03.043)

Potthoff MJ, Wu H, Arnold MA, Shelton JM, Backs J, McAnally J, Richardson JA, Bassel-Duby R \& Olson EN 2007 Histone deacetylase degradation and MEF2 activation promote the formation of slow-twitch myofibers. Journal of Clinical Investigation 117 2459-2467. (doi:10.1172/JCI31960)

Savage DB, Petersen KF \& Shulman GI 2007 Disordered lipid metabolism and the pathogenesis of insulin resistance. Physiological Reviews 87 507-520. (doi:10.1152/physrev.00024.2006)

Takigawa-Imamura H, Sekine T, Murata M, Takayama K, Nakazawa K \& Nakagawa J 2003 Stimulation of glucose uptake in muscle cells by prolonged treatment with scriptide, a histone deacetylase inhibitor. Bioscience, Biotechnology, and Biochemistry 67 1499-1506. (doi:10. 1271/bbb.67.1499)

Vega RB, Matsuda K, Oh J, Barbosa AC, Yang X, Meadows E, McAnally J, Pomajzl C, Shelton JM, Richardson JA et al. 2004 Histone deacetylase 4 controls chondrocyte hypertrophy during skeletogenesis. Cell 119 555-566. (doi:10.1016/j.cell.2004.10.024)

Xing W, Zhang TC, Cao D, Wang Z, Antos CL, Li S, Wang Y, Olsen EN \& Wang DZ 2006 Myocardin induces cardiomyocyte hypertrophy. Circulation Research 98 1089-1097. (doi:10.1161/01.RES. $0000218781.23144 .3 \mathrm{e})$

Zhang CL, McKinsey TA \& Olsen EN 2001 The transcriptional corepressor MITR is a signal responsive inhibitor of myogenesis. PNAS 98 7354-7359. (doi:10.1073/pnas.131198498)

Zhang CL, McKinsey TA \& Olson EN 2002 Association of class II histone deacetylases with heterochromatin protein 1: potential role for histone methylation in control of muscle differentiation. Molecular and Cellular Biology 22 7302-7312. (doi:10.1128/MCB.22. 20.7302-7312.2002)

Received in final form 11 September 2012

Accepted 18 September 2012

Made available online as an Accepted Preprint

18 September 2012 